\title{
Estimates of Certain Integral Inequalities on Time Scales
}

\author{
Deepak B. Pachpatte \\ Department of Mathematics, Dr. B.A.M. University, Aurangabad, Maharashtra 431004, India
}

Correspondence should be addressed to Deepak B. Pachpatte; pachpatte@gmail.com

Received 13 August 2012; Accepted 22 October 2012

Academic Editor: Abdul Hamid Kara

Copyright (c) 2013 Deepak B. Pachpatte. This is an open access article distributed under the Creative Commons Attribution License, which permits unrestricted use, distribution, and reproduction in any medium, provided the original work is properly cited.

The main objective of this paper is to establish explicit bounds on certain integral inequalities on time scales, which can be used as tools in the study of certain classes of integral equations on time scales. Some applications of our results are also given.

\section{Introduction}

Recently, many authors have studied various aspects of dynamic inequalities on time scales using various techniques [1-4]. In this paper, we obtain explicit bounds on certain integral inequalities on time scales. In the present paper we offer some fundamental dynamic integral inequalities on time scales which can be used as tools for handling the qualitative behavior of solutions of certain dynamic equations on time scales. Excellent information about introduction to time scales can be found in $[5,6]$. In what follows, $\mathbb{R}$ denotes the set of real numbers, $\mathbb{Z}$ the set of integers, and $\mathbb{T}$ denotes arbitrary time scales. Now following $[3,4]$ we give some basic definitions about calculus on time scales in two variables.

We say that $f: \mathbb{T} \rightarrow \mathbb{R}$ is rd-continuous provided $f$ is continuous at each right-dense point of $\mathbb{T}$ and has a finite left sided limit at each left-dense point of $\mathbb{T}$ and will be denoted by $C_{\text {rd }}$. Let $\mathbb{T}_{1}$ and $\mathbb{T}_{2}$ be two time scales with at least two points and consider the time scales intervals $\overline{\mathbb{T}}_{1}=\left[x_{0}, \infty\right) \cap \mathbb{T}_{1}$ and $\overline{\mathbb{T}}_{2}=\left[y_{0}, \infty\right) \cap \mathbb{T}_{2}$ for $x_{0} \in \mathbb{T}_{1}$ and $y_{0} \in \mathbb{T}_{2}$ and $\Omega=\mathbb{T}_{1} \times \mathbb{T}_{2}$. Let $\sigma_{1}, \rho_{1}, \Delta_{1}$ and $\sigma_{2}, \rho_{2}, \Delta_{2}$ denote the forward jump operators, backward jump operators and the delta differentiation operator, respectively, on $\mathbb{T}_{1}$ and $\mathbb{T}_{2}$. Let $a<b$ are points in $\mathbb{T}_{1}, c<d$ are points in $\mathbb{T}_{2},[a, b)$ is the half closed bounded interval in $\mathbb{T}_{1}$, and $[c, d)$ is the half closed bounded interval in $\mathbb{T}_{2}$.

We say that a real-valued function $f$ on $\mathbb{T}_{1} \times \mathbb{T}_{2}$ at $\left(t_{1}, t_{2}\right) \in \overline{\mathbb{T}}_{1} \times \overline{\mathbb{T}}_{2}$ has a $\Delta_{1}$ partial derivative $f^{\Delta_{1}}\left(t_{1}, t_{2}\right)$ with respect to $t_{1}$ if for each $\epsilon>0$ there exists a neighborhood $U_{t_{1}}$ of $t_{1}$ such that

$$
\begin{aligned}
& \left|f\left(\sigma_{1}\left(t_{1}\right), t_{2}\right)-f\left(s, t_{2}\right)-f^{\Delta_{1}}\left(t_{1}, t_{2}\right)\left(\sigma_{1}\left(t_{1}\right)-s\right)\right| \\
& \quad \leq \varepsilon\left|\sigma_{1}\left(t_{1}\right)-s\right|
\end{aligned}
$$

for all $s \in U_{t_{1}}$. We say that $f$ on $\mathbb{T}_{1} \times \mathbb{T}_{2}$ at $\left(t_{1}, t_{2}\right) \in \overline{\mathbb{T}}_{1} \times \overline{\mathbb{T}}_{2}$ has a $\Delta_{2}$ partial derivative $f^{\Delta_{2}}\left(t_{1}, t_{2}\right)$ with respect to $t_{2}$ if for each $\eta>0$ there exists a neighborhood $U_{t_{2}}$ of $t_{2}$ such that

$$
\begin{aligned}
& \left|f\left(t_{1}, \sigma_{2}\left(t_{2}\right)\right)-f\left(t_{1}, l\right)-f^{\Delta_{2}}\left(t_{1}, t_{2}\right)\left(\sigma_{2}\left(t_{2}\right)-l\right)\right| \\
& \quad \leq \eta\left|\sigma_{2}\left(t_{2}\right)-l\right|
\end{aligned}
$$

for all $l \in U_{t_{2}}$. The function $f$ is called rd-continuous in $t_{2}$ if for every $\alpha_{1} \in \mathbb{T}_{1}$, the function $f\left(\alpha_{1}, t_{2}\right)$ is rd-continuous on $\mathbb{T}_{2}$. The function $f$ is called rd-continuous in $t_{1}$ if for every $\alpha_{2} \in \mathbb{T}_{2}$ the function $f\left(t_{1}, \alpha_{2}\right)$ is rd-continuous on $\mathbb{T}_{1}$.

We require that the following lemma proved in $[1,2]$ holds.

Lemma 1. Let $u, f \in C_{r d}(\mathbb{T}, \mathbb{R}), k(t, s), k^{\Delta_{1}}(t, s) \in C_{r d}(\Omega, \mathbb{R})$ and $c$ nonnegative constants. If

$$
u(t) \leq c+\int_{t_{0}}^{t} f(s)\left[u(s)+\int_{s_{0}}^{s} k(s, \tau) u(\tau) \Delta \tau\right] \Delta s,
$$


for $t \in \mathbb{T}$, then

$$
u(t) \leq c\left[1+\int_{t_{0}}^{t} f(s) e_{f+A}\left(s, s_{0}\right) \Delta s\right],
$$

for $t \in \mathbb{T}$, where

$$
A(t)=k(t, \sigma(t))+\int_{t_{0}}^{t} k^{\Delta_{1}}(t, \eta) \Delta \eta,
$$

for $t \in \mathbb{T}$.

Lemma 2 (see [1, Lemma]). Let $u, a, f \in C_{\mathrm{rd}}^{\prime}\left(\overline{\mathbb{T}}_{1} \times \overline{\mathbb{T}}_{2}, \mathbb{R}_{+}\right)$ and $a$ is nondecreasing in each of its variables. If

$$
u(x, y) \leq a(x, y)+\int_{x_{0}}^{x} \int_{y_{0}}^{y} f(s, t) u(s, t) \Delta t \Delta s,
$$

for $(x, y) \in \overline{\mathbb{T}}_{1} \times \overline{\mathbb{T}}_{2}$, then

$$
u(x, y) \leq a(x, y)+e_{y_{0}}^{y} f(x, t) \Delta t\left(x, x_{0}\right),
$$

for $(x, y) \in \overline{\mathbb{T}}_{1} \times \overline{\mathbb{T}}_{2}$.

\section{Main Results}

Our main results are given in the following theorems.

Theorem 3. Let $u, f, a \in C_{\mathrm{rd}}\left(\mathbb{T}, R_{+}\right), k(t, s), k^{\Delta}(t, s) \in$ $C_{\mathrm{rd}}\left(\Omega, R_{+}\right)$. If

$$
u(t) \leq a(t)+\int_{t_{0}}^{t} f(s)\left[u(s)+\int_{s_{0}}^{s} k(s, \tau) u(\tau) \Delta \tau\right] \Delta s,
$$

for $t \in \mathbb{T}$, then

$$
u(t) \leq a(t)+h(t)\left[1+\int_{t_{0}}^{t} f(s) e_{f+A}\left(s, s_{0}\right) \Delta s\right],
$$

for $t \in \mathbb{T}$, where

$$
h(t)=\int_{t_{0}}^{t} f(s)\left[a(s)+\int_{s_{0}}^{s} k(s, \tau) a(\tau) \Delta \tau\right] \Delta s,
$$

for $t \in \mathbb{T}$ and $A(t)$ defined by (5).

Proof. Define a function $z(t)$ by

$$
z(t)=\int_{t_{0}}^{t} f(s)\left[u(s)+\int_{s_{0}}^{s} k(s, \tau) u(\tau) \Delta \tau\right] \Delta s,
$$

then from (8), $u(t) \leq a(t)+z(t)$, and using this in (11) we get

$$
\begin{aligned}
z(t) & \leq \int_{t_{0}}^{t} f(s)\left[a(s)+z(s)+\int_{s_{0}}^{s} k(s, \tau)(a(\tau)+z(\tau)) \Delta \tau\right] \Delta s \\
& =h(t)+\int_{t_{0}}^{t} f(s)\left[z(s)+\int_{s_{0}}^{s} k(s, \tau) z(\tau) \Delta \tau\right] \Delta s,
\end{aligned}
$$

where $h(t)$ is defined by (10). Clearly $h(t)$ is nonnegative, rd-continuous and nondecreasing in $t, t \in \mathbb{T}$ for $a(t)$ nondecreasing. First we assume that $h(t)>0$ for $t \in \mathbb{T}$. From (12), we have

$$
\frac{z(t)}{h(t)} \leq 1+\int_{t_{0}}^{t} f(s)\left[\frac{z(s)}{h(t)}+\int_{s_{0}}^{s} k(s, \tau) \frac{z(\tau)}{h(\tau)} \Delta \tau\right] \Delta s .
$$

Now as an application of Lemma 1 we have

$$
\frac{z(t)}{h(t)} \leq\left[1+\int_{t_{0}}^{t} f(s) e_{f+A}\left(s, s_{0}\right) \Delta s\right] .
$$

The desired inequality (9) follows from (14) and the fact that $u(t) \leq a(t)+z(t)$. If $h(t) \geq 0$, we carry out the above procedure with $h(t)+\epsilon$ instead of $h(t)$, where $\epsilon>0$ is an arbitrary small constants, and then subsequently pass to the limit as $\epsilon \rightarrow 0$ to obtain (9). The following theorem deals with two independent versions of inequalities established in Theorem 3.

Theorem 4. Let $u, f \in C_{\mathrm{rd}}\left(\Omega, R_{+}\right), k(x, y, s, t), k^{\Delta_{1}}(x, y, s, t)$, $k^{\Delta_{2}}(x, y, s, t), k^{\Delta_{2} \Delta_{1}}(x, y, s, t) \in C_{\mathrm{rd}}\left(\Omega \times \Omega, R_{+}\right)$, and $c$ be a nonnegative constants. If

$$
\begin{aligned}
u(x, y) \leq & c+\int_{x_{0}}^{x} \int_{y_{0}}^{y} f(s, t) \\
& \times\left[u(s, t)+\int_{s_{0}}^{s} \int_{t_{0}}^{t} k(s, t, \tau, \xi) u(\tau, \xi) \Delta \xi \Delta \tau\right] \Delta t \Delta s,
\end{aligned}
$$

for $(x, y) \in \Omega$, then

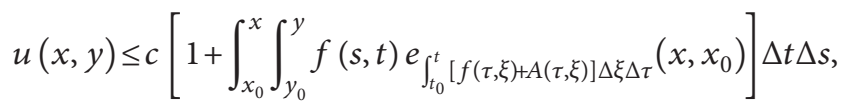

where

$$
\begin{aligned}
A(x, y)= & k(x, y, \sigma(x), \sigma(y))+\int_{x_{0}}^{x} k^{\Delta_{1}}(x, y, \tau, \sigma(y)) \Delta \tau \\
& +\int_{y_{0}}^{y} k^{\Delta_{1}}(x, y, \sigma(x), \eta) \Delta \eta \\
& +\int_{x_{0}}^{x} \int_{y_{0}}^{y} k^{\Delta_{2} \Delta_{1}}(x, y, \tau, \eta) \Delta \eta \Delta \tau,
\end{aligned}
$$

for $(x, y) \in \Omega$.

Proof. Let $c>0$ and define a function $z(x, y)$ by the righthand side of (15); then $z\left(x_{0}, y\right)=z\left(x, y_{0}\right)=c, u(x, y) \leq$ $z(x, y)$, and

$$
\begin{aligned}
& z^{\Delta_{2} \Delta_{1}}(x, y) \\
& \quad=f(x, y)\left[u(x, y)+\int_{x_{0}}^{x} \int_{y_{0}}^{y} k(x, y, \tau, \xi) u(\tau, \xi) \Delta \xi \Delta \tau\right] \\
& \quad \leq f(x, y)\left[z(x, y)+\int_{x_{0}}^{x} \int_{y_{0}}^{y} k(x, y, \tau, \xi) z(\tau, \xi) \Delta \xi \Delta \tau\right] .
\end{aligned}
$$


Define a function $v(x, y)$ by

$$
v(x, y)=z(x, y)+\int_{x_{0}}^{x} \int_{y_{0}}^{y} k(x, y, \tau, \xi) z(\tau, \xi) \Delta \xi \Delta \tau,
$$

then $v\left(x_{0}, y\right)=z\left(x_{0}, y\right)=c, v\left(x, y_{0}\right)=z\left(x, y_{0}\right)=c$, $z(x, y) \leq v(x, y), z^{\Delta_{1} \Delta_{2}}(x, y) \leq f(x, y) v(x, y), v(x, y)$ is nondecreasing for $(x, y) \in \Omega$, and

$$
\begin{aligned}
v^{\Delta_{1} \Delta_{2}}(x, y)= & z^{\Delta_{1} \Delta_{2}}(x, y)+k(x, y, \sigma(x), \sigma(y)) z(x, y) \\
& +\int_{x_{0}}^{x} k^{\Delta_{1}}(x, y, \tau, \sigma(y)) z(\tau, y) \Delta \tau \\
& +\int_{y_{0}}^{y} k^{\Delta_{2}}(x, y, \sigma(x), \xi) z(x, \xi) \Delta \xi \\
& +\int_{x_{0}}^{x} \int_{y_{0}}^{y} k^{\Delta_{2} \Delta_{1}}(x, y, \tau, \xi) z(\tau, \xi) \Delta \xi \Delta \tau \\
\leq & f(x, y) v(x, y)+k(x, y, \sigma(x), \sigma(y)) v(x, y) \\
& +\int_{x_{0}}^{x} k^{\Delta_{1}}(x, y, \tau, \sigma(y)) v(\tau, y) \Delta \tau \\
& +\int_{y_{0}}^{y} k^{\Delta_{2}}(x, y, \sigma(x), \xi) v(x, \xi) \Delta \xi \\
& +\int_{x_{0}}^{x} \int_{y_{0}}^{y} k^{\Delta_{2} \Delta_{1}}(x, y, \tau, \xi) v(\tau, \xi) \Delta \xi \Delta \tau \\
\leq & |f(x, y)+A(x, y)| v(x, y),
\end{aligned}
$$

where $A(x, y)$ is defined by (17).

Using the fact that $z(x, y)>0, z^{\Delta_{1}}(x, y) \geq 0, z^{\Delta_{2}}(x, y) \geq$ 0 , and we have

$$
\frac{v^{\Delta_{2} \Delta_{1}}(x, y)}{v(x, y)} \leq[f(x, y)+A(x, y)]+\frac{v^{\Delta_{1}}(x, y) v^{\Delta_{2}}(x, y)}{v(x, y) v(x, \sigma(y))} .
$$

Now keeping $x$ fixed and delta integrating with respect to $t$ from $y_{0}$ to $y$, we obtain estimates

$$
\frac{v^{\Delta_{1}}(x, y)}{v(x, y)} \leq \int_{y_{0}}^{y}[f(x, \xi)+A(x, y)] \Delta_{2} \xi,
$$

keeping $y$ fixed in (22) and delta integrating $s$ from $x_{0}$ to $x$, we get

$$
v(x, y) \leq c+\int_{x_{0}}^{x} \int_{y_{0}}^{y}[f(\tau, \xi)+A(\tau, \xi)] v(x, y) \Delta_{2} \xi \Delta_{1} \tau .
$$

Then Lemma 2 implies

$$
v(x, y) \leq c+e \int_{y_{0}}^{y}[f(\tau, \xi)+A(\tau, \xi)] \Delta \xi\left(x, x_{0}\right) .
$$

Using (24) in (18) and integrating the resulting inequality from 0 to $y$ and then from 0 to $x$ for $(x, y) \in \Omega$, we get

$$
z(x, y) \leq c\left[1+\int_{x_{0}}^{x} \int_{y_{0}}^{y} f(s, \tau) e_{\int_{t_{0}}^{t}[f(\tau, \xi)+A(\tau, \xi)] \Delta \xi \Delta \tau}\left(s, s_{0}\right) \Delta t \Delta s\right] .
$$

Using (25) in $u(x, y) \leq z(x, y)$, we get the required inequality in (16).

Theorem 5. Let $u, f, a \in C_{\mathrm{rd}}\left(\Omega \times \Omega, R_{+}\right)$and $k(x, y, s, t)$, $k^{\Delta_{1}}(x, y, s, t), k^{\Delta_{2}}(x, y, s, t), k^{\Delta_{2} \Delta_{1}}(x, y, s, t) \in C_{\mathrm{rd}}\left(\Omega, R_{+}\right)$. If

$u(x, y)$

$$
\begin{aligned}
\leq a(x, y)+ & \int_{x_{0}}^{x} \int_{y_{0}}^{y} f(s, t) \\
& \times\left[u(s, t)+\int_{s_{0}}^{s} \int_{t_{0}}^{t} k(s, t, \tau, \xi) u(\tau, \xi) \Delta \xi \Delta \tau\right] \Delta t \Delta s,
\end{aligned}
$$

For $(x, y) \in \Omega$, then

$$
\begin{aligned}
& u(x, y) \\
& \leq a(x, y)+\bar{h}(x, y) \\
& \quad \times\left[1+\int_{x_{0}}^{x} \int_{y_{0}}^{y} f(s, t) e_{\int_{0}}^{t}[f(\tau, \xi)+A(\tau, \xi)] \Delta \xi \Delta \tau\right. \\
& \left.\quad\left(x, x_{0}\right)\right] \Delta t \Delta s,
\end{aligned}
$$

where

$$
\begin{aligned}
& \bar{h}(x, y) \\
& =\int_{x_{0}}^{x} \int_{y_{0}}^{y} f(s, t) \\
& \quad \times\left[a(s, t)+\int_{s_{0}}^{s} \int_{t_{0}}^{t} k(s, t, \sigma(x), \xi) a(\tau, \xi) \Delta \xi \Delta \tau\right] \Delta t \Delta s,
\end{aligned}
$$

for $(x, y) \in \Omega$ and $A(x, y)$ as defined in (17).

Proof. Define a function $z(x, y)$ by the right-hand side of (26)

$$
\begin{aligned}
z(x, y) \leq & \int_{x_{0}}^{x} \int_{y_{0}}^{y} f(s, t) \\
& \times\left[u(s, t)+\int_{s_{0}}^{s} \int_{t_{0}}^{t} k(s, t, \tau, \xi) u(\tau, \xi) \Delta \xi \Delta \tau\right] \Delta t \Delta s,
\end{aligned}
$$


then from (26) we have $u(x, y) \leq a(x, y)+z(x, y)$, and using this in (28) we get

$$
\begin{aligned}
& z(x, y) \\
& \leq \int_{x_{0}}^{x} \int_{y_{0}}^{y} f(s, t)[a(s, t)+z(s, t) \\
& +\int_{s_{0}}^{s} \int_{t_{0}}^{t} k(s, t, \tau, \xi) \\
& \times[a(\tau, \xi)+z(\tau, \xi)] \Delta \xi \Delta \tau] \Delta t \Delta s \\
& \leq \bar{h}(x, y)+\int_{x_{0}}^{x} \int_{y_{0}}^{y} f(s, t)\left[z(s, t)+\int_{s_{0}}^{s} \int_{t_{0}}^{t} k(s, t, \tau, \xi)\right. \\
& \times z(\tau, \xi) \Delta \xi \Delta \tau] \Delta t \Delta s
\end{aligned}
$$

where $\bar{h}(x, y)$ is defined by (28). Here $\bar{h}(x, y)$ nondecreasing for $(x, y) \in \Omega$, then from (30) we have

$$
\begin{aligned}
& \frac{z(x, y)}{\overline{\bar{h}}(x, y)} \\
& \quad \leq 1+\int_{x_{0}}^{x} \int_{y_{0}}^{y} f(s, t) \\
& \quad \times\left[\frac{z(s, t)}{\bar{h}(s, t)}+\int_{s_{0}}^{s} \int_{t_{0}}^{t} k(s, t, \tau, \xi) \frac{z(\tau, \xi)}{\bar{h}(\tau, \xi)} \Delta \xi \Delta \tau\right] \Delta t \Delta s .
\end{aligned}
$$

Now as an application of inequality in Theorem 3 to (31) we get

$$
\frac{z(x, y)}{\bar{h}(x, y)} \leq\left[1+\int_{x_{0}}^{x} \int_{y_{0}}^{y} f(s, t) e_{[f(\tau, \xi)+A(\tau, \xi)] \Delta \xi \Delta \tau}\left(x, x_{0}\right)\right] \Delta t \Delta s .
$$

The required inequality in (27) follows from (32) and using the fact that $u(x, y) \leq a(x, y)+z(x, y)$.

\section{Applications}

In this section, we give application of inequality in Theorem 4 to study certain properties of solution of nonlinear partial integrodifferential equation on time scales,

$$
\begin{aligned}
& u^{\Delta_{2} \Delta_{1}}(x, y) \\
& \quad=F\left(x, y, u(x, y), \int_{x_{0}}^{x} \int_{y_{0}}^{y} h(x, y, \tau, \xi, u(\tau, \xi)) \Delta \xi \Delta \tau\right),
\end{aligned}
$$

with the initial boundary condition

$$
\begin{gathered}
u\left(x, y_{0}\right)=\alpha_{1}(x), \\
u\left(x_{0}, y\right)=\alpha_{2}(y), \\
\alpha_{1}(0)=\alpha_{2}(0)=0,
\end{gathered}
$$

where $u \in C_{\mathrm{rd}}\left(\Omega, R_{+}\right), h \in C_{\mathrm{rd}}\left(\Omega \times R, R_{+}\right)$, and $F \in C_{\mathrm{rd}}(\Omega \times$ $\left.\Omega, R_{+}\right)$.

The following theorem deals with the estimate on the solution of (33)-(34).

Theorem 6. Assume that

$$
\begin{gathered}
|h(x, y, s, t, u(s, t))| \leq k(x, y, s, t)|u(s, t)|, \\
|F(x, y, u, v)| \leq f(x, y)[|u|+|v|], \\
\left|\alpha_{1}(x)+\alpha_{2}(y)\right| \leq c
\end{gathered}
$$

where $k, f$, and $c$ are as defined in (9). If $u(x, y),(x, y) \in \Omega$ is any solution of (33)-(34), then

$$
\begin{aligned}
& |u(x, y)| \\
& \quad \leq c\left[1+\int_{x_{0}}^{x} \int_{y_{0}}^{y} f(s, t) e_{\int_{t_{0}}^{t}}[f(\tau, \xi)+A(\tau, \xi)] \Delta t\right. \\
& \left.\quad\left(s, s_{0}\right) \Delta t \Delta s\right]
\end{aligned}
$$

for $(x, y) \in \Omega$, where $A(x, y)$ is defined by (17).

Proof. The solution $u(x, y)$ of (33)-(34) can be written as

$$
\begin{aligned}
u(x, y)= & \alpha_{1}(x)+\alpha_{2}(y) \\
& +\int_{x_{0}}^{x} \int_{y_{0}}^{y} F(s, t, u(s, t), \\
& \left.\int_{s_{0}}^{s} \int_{t_{0}}^{t} h(s, t, \tau, \xi, u(\tau, \xi)) \Delta \xi \Delta \tau\right) \Delta t \Delta s .
\end{aligned}
$$

Using (35) in (37), we have

$$
\begin{aligned}
& |u(x, y)| \\
& \leq c+\int_{x_{0}}^{x} \int_{y_{0}}^{y} f(s, t) \\
& \times\left[|u(s, t)|+\left(\int_{s_{0}}^{s} \int_{t_{0}}^{t} k(s, t, \tau, \xi,|u(\tau, \xi)|)\right.\right. \\
& \times \Delta \xi \Delta \tau)] \Delta t \Delta s .
\end{aligned}
$$

Now an application of inequality in Theorem 4 yields the estimate in (36). (34).

In the next result, we give uniqueness of solutions of (33)- 
Theorem 7. Suppose that the functions h, F in (33) satisfy the conditions

$$
\begin{gathered}
\left|h\left(x, y, s, t, u_{1}\right)-h\left(x, y, s, t, u_{2}\right)\right| \leq k(x, y, s, t)\left|u_{1}-u_{2}\right| \\
\left|F\left(x, y, u_{1}, u_{2}\right)-F\left(x, y, v_{1}, v_{2}\right)\right| \\
\leq f(x, y)\left[\left|u_{1}-v_{1}\right|+\left|u_{2}-v_{2}\right|\right]
\end{gathered}
$$

where $k$ and $f$ are as in Theorem 4. Then the problem (33)-(34) has at most one solution on $\Omega$.

Proof. Let $u_{1}(x, y)$ and $u_{2}(x, y)$ be two solutions of (33)-(34) on $\Omega$, then we have

$$
\begin{aligned}
& u_{1}(x, y)-u_{2}(x, y) \\
&=\int_{x_{0}}^{x} \int_{y_{0}}^{y}[ F\left(s, t, u_{1}(s, t), \int_{s_{0}}^{s} \int_{t_{0}}^{t} h\left(s, t, \tau, \xi, u_{1}(\tau, \xi)\right) \Delta \xi \Delta \tau\right) \\
&-F\left(s, t, u_{2}(s, t), \int_{s_{0}}^{s} \int_{t_{0}}^{t} h\left(s, t, \tau, \xi, u_{2}(\tau, \xi)\right)\right. \\
&\times \Delta \xi \Delta \tau)] \Delta t \Delta s .
\end{aligned}
$$

From (39) and (40), we have

$$
\begin{aligned}
& \left|u_{1}(x, y)-u_{2}(x, y)\right| \\
& \leq \int_{x_{0}}^{x} \int_{y_{0}}^{y}\left[f(s, t)\left|u_{1}(s, t)-u_{2}(s, t)\right|\right. \\
& \quad+\int_{s_{0}}^{s} \int_{t_{0}}^{t} k(s, t, \tau, \xi)\left|u_{1}(\tau, \xi)-u_{2}(\tau, \xi)\right| \\
& \times \Delta \xi \Delta \tau] \Delta t \Delta s .
\end{aligned}
$$

As an application of inequality in Theorem 4 with $c=0$ yields $\left|u_{1}(x, y)-u_{2}(x, y)\right| \leq 0$, therefore $u_{1}(x, y)=u_{2}(x, y)$; that is, there is at most one solution of (33)-(34) on $\Omega$.

\section{References}

[1] R. A. C. Ferreira and D. F. M. Torres, "Some linear and nonlinear integral inequalities on time scales in two independent variables," Nonlinear Dynamics and Systems Theory, vol. 9, no. 2, pp. 161-169, 2009.

[2] D. B. Pachpatte, "On a nonlinear dynamic integrodifferential equation on time scales," Journal of Applied Analysis, vol. 16, pp. 279-294, 2010.

[3] W. Liu, Q. A. Ngo, and W. Chen, "Ostrowski type inequalities on time scales for double integrals," Acta Applicandae Mathematicae, vol. 110, no. 1, pp. 477-497, 2010.

[4] U. M. Özkan and H. Yildirim, "Ostrowski type inequality for double integrals on time scales," Acta Applicandae Mathematicae, vol. 110, no. 1, pp. 283-288, 2010.

[5] M. Bohner and A. Peterson, Dynamic Equations on Time Scales, Birkhauser, Berlin, Germany, 2001.
[6] M. Bohner and A. Peterson, Advances in Dynamic Equations on Time Scales, Birkhauser, Berlin, Germany, 2003. 


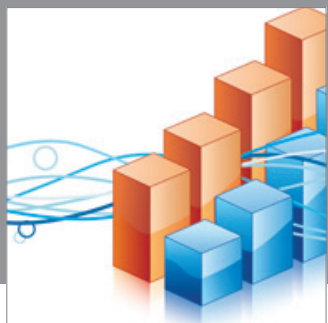

Advances in

Operations Research

mansans

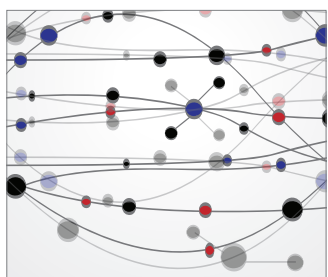

The Scientific World Journal
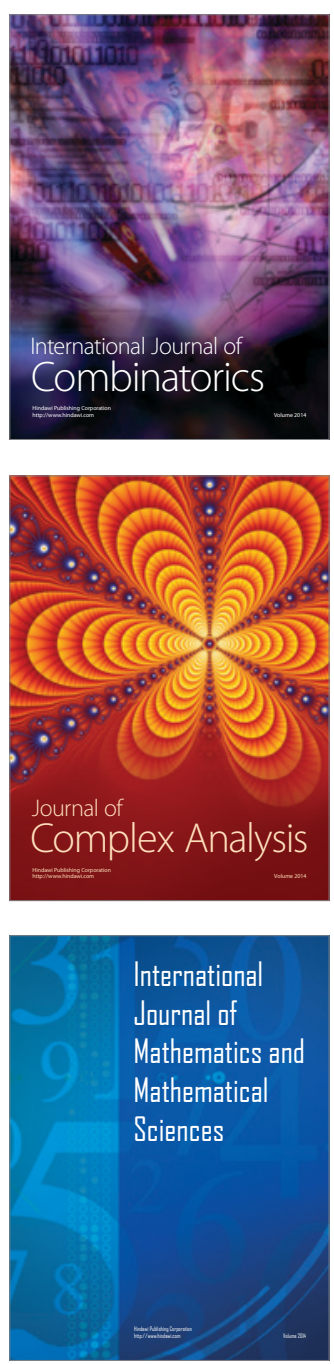
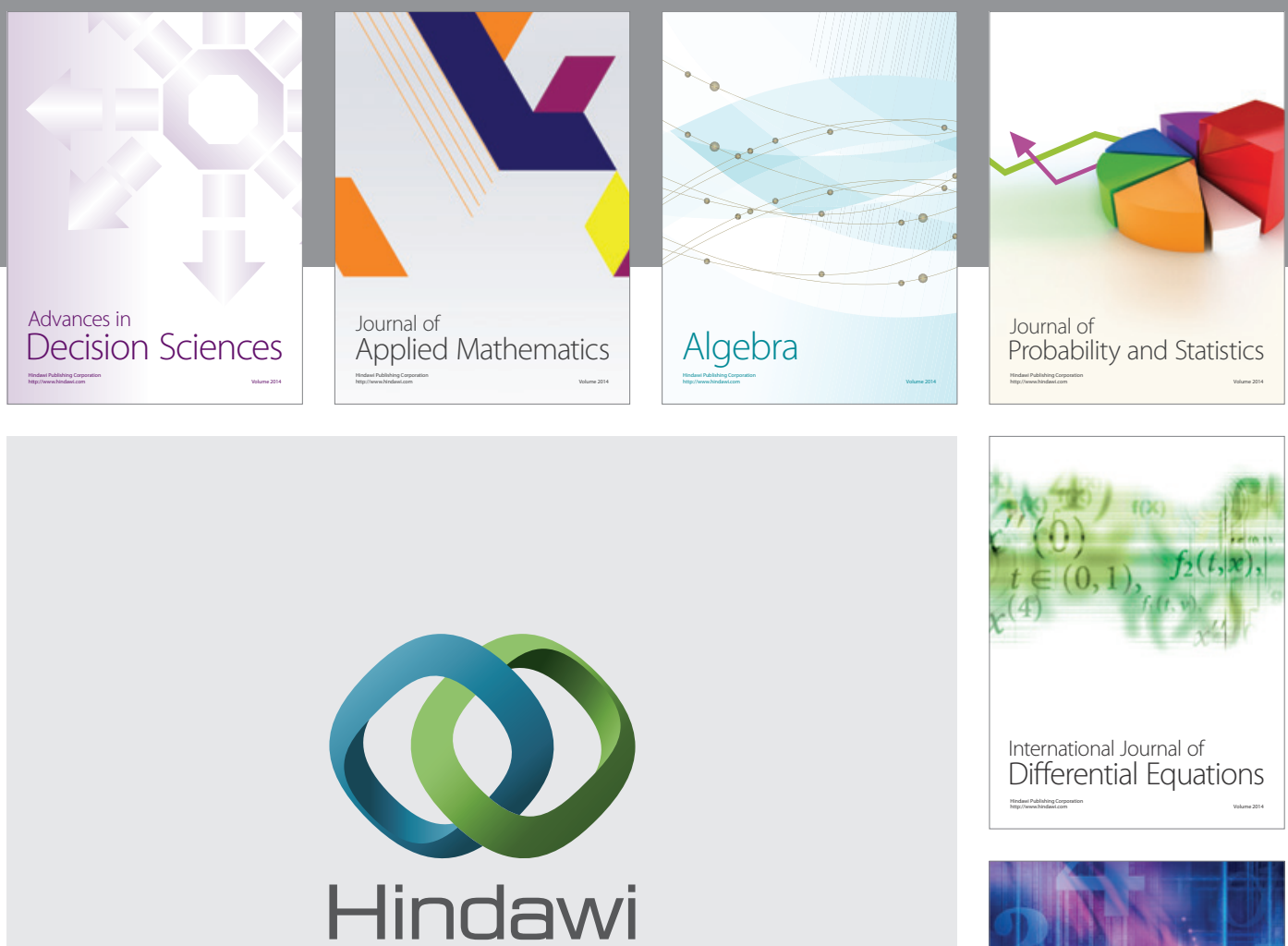

Submit your manuscripts at http://www.hindawi.com
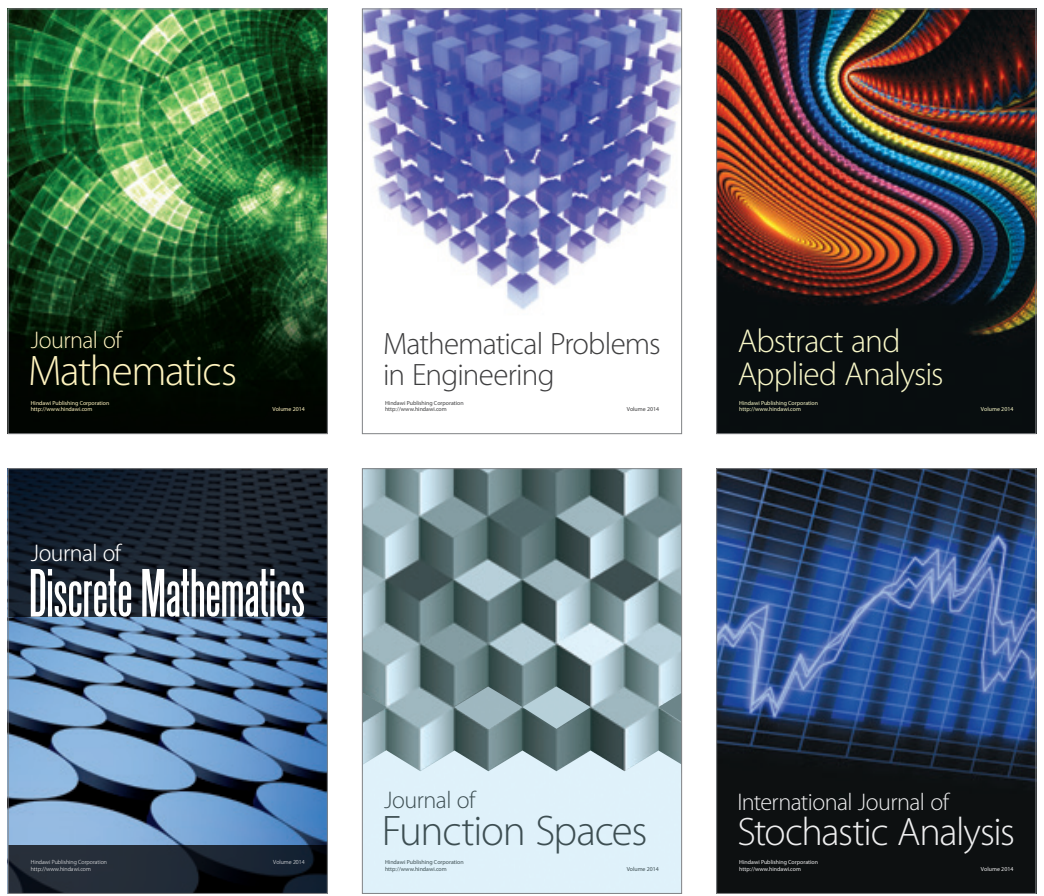

Journal of

Function Spaces

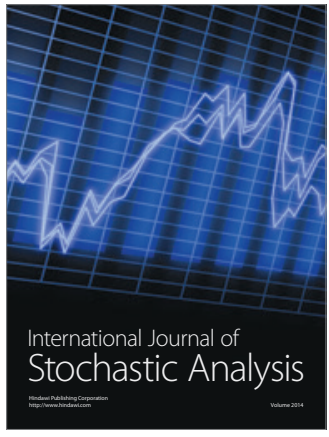

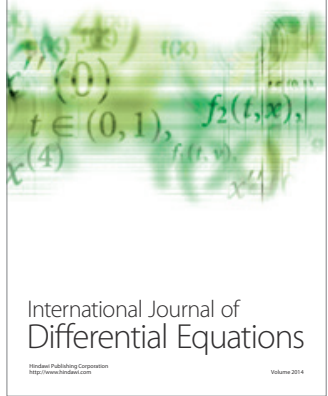
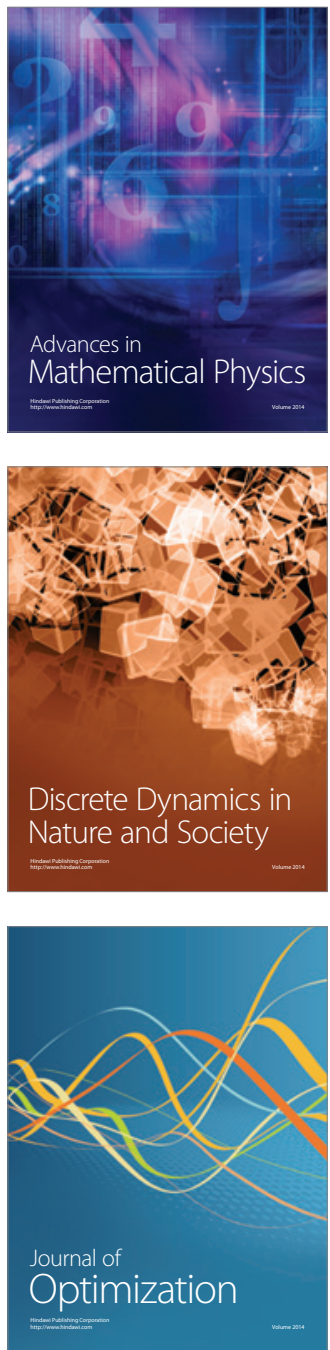